TRANSACTIONS OF THE

AMERICAN MATHEMATICAL SOCIETY

Volume 358, Number 1, Pages 147-165

S 0002-9947(04)03609-8

Article electronically published on December 28, 2004

\title{
THE LIMITING ABSORPTION PRINCIPLE FOR THE TWO-DIMENSIONAL INHOMOGENEOUS ANISOTROPIC ELASTICITY SYSTEM
}

\author{
GEN NAKAMURA AND JENN-NAN WANG
}

\begin{abstract}
In this work we establish the limiting absorption principle for the two-dimensional steady-state elasticity system in an inhomogeneous anisotropic medium. We then use the limiting absorption principle to prove the existence of a radiation solution to the exterior Dirichlet or Neumann boundary value problems for such a system. In order to define the radiation solution, we need to impose certain appropriate radiation conditions at infinity. It should be remarked that even though in this paper we assume that the medium is homogeneous outside of a large domain, it still preserves anisotropy. Thus the classical Kupradze's radiation conditions for the isotropic system are not suitable in our problem and new radiation conditions are required. The uniqueness of the radiation solution plays a key role in establishing the limiting absorption principle. To prove the uniqueness of the radiation solution, we make use of the unique continuation property, which was recently obtained by the authors. The study of this work is motivated by related inverse problems in the anisotropic elasticity system. The existence and uniqueness of the radiation solution are fundamental questions in the investigation of inverse problems.
\end{abstract}

\section{INTRODUCTION}

In this paper we investigate the uniqueness and existence of the radiation solution for the two-dimensional steady-state elasticity system with inhomogeneous anisotropic medium in an exterior domain. The main point is to establish the limiting absorption principle for the related elastic operator. The limiting absorption principle has been studied for many differential operators arising from mathematical physics. We refer to [10] and references therein for a more detailed discussion of recent development.

Let $\Omega$ be an open exterior domain in $\mathbb{R}^{2}$ with smooth boundary $\partial \Omega$ such that $\mathbb{R}^{2} \backslash \bar{\Omega} \subset\left\{|x|<R_{0}\right\}$ for some $R_{0}>0$. Here we do not exclude the case when $\Omega=\mathbb{R}^{2}$. In this paper we consider the steady-state inhomogeneous anisotropic elasticity system in $\Omega$

$$
\nabla \cdot(C(x) \nabla u)+\omega^{2} u=f \quad \text { in } \Omega, \quad \omega>0,
$$

Received by the editors September 15, 2003 and, in revised form, January 5, 2004.

2000 Mathematics Subject Classification. Primary 35J55, 74G25, 74G30; Secondary 74B05, $74 \mathrm{E} 10$.

Key words and phrases. Limiting absorption principle, anisotropic elasticity system, radiation conditions.

The first author was partially supported by Grant-in-Aid for Scientific Research (B)(2) (No.14340038) of the Japan Society for the Promotion of Science.

The second author was partially supported by the National Science Council of Taiwan. 
where $C(x)=\left(C_{i j k l}(x)\right)$ is the elastic tensor and $u=\left[u_{1}, u_{2}\right]^{t}$ is the displacement vector. Here and further on we use the convention that all Roman indices are set to be from 1 to 2 . Also we would like to call to the reader's attention that " " denotes the imaginary number $\sqrt{-1}$. In (1.1) and what follows, we interpret $(\nabla u)_{k l}=\partial_{l} u_{k}$, $(\nabla \cdot H)_{i}=\sum_{j} \partial_{j} h_{i j}$ for any matrix function $H=\left(h_{i j}\right)$, and

$$
(C H)_{i j}=\sum_{k l} C_{i j k l} h_{k l} .
$$

The elastic tensor $C(x)$ is assumed to satisfy the full symmetry properties

$$
C_{i j k l}(x)=C_{j i k l}(x)=C_{k l i j}(x) \quad \forall i, j, k, l \text { and } x \in \Omega
$$

and the strong convexity condition, i.e., there exists a $\delta>0$ such that for all $x \in \Omega$

$$
C(x) E \cdot E \geq \delta|E|^{2}
$$

for any symmetric matrix $E$. Furthermore, we suppose that for large $|x|$ the medium is homogeneous, namely, $C(x)=C$ for $|x| \geq R$ with some $R>R_{0}$. In order to avoid clumsy notations, we will use $C$ (or $C_{i j k l}$ ) and $C(x)$ (or $C_{i j k l}(x)$ ) to denote the homogeneous and the inhomogeneous tensor, respectively. Note that we do not assume that the constant elastic tensor $C$ is isotropic. We recall here that the elastic tensor $C$ is called isotropic, if $C_{i j k l}=\lambda \delta_{i j} \delta_{k l}+\mu\left(\delta_{j l} \delta_{i k}+\delta_{i l} \delta_{j k}\right)$, where $\lambda$ and $\mu$ are Lamé parameters. To formulate the boundary value problem related to (1.1), we impose on the boundary $\partial \Omega$ the Dirichlet condition

$$
\left.u\right|_{\partial \Omega}=0,
$$

or the Neumann condition

$$
\left.T(D, n) u\right|_{\partial \Omega}=\left.\sigma n\right|_{\partial \Omega}=0,
$$

where $\sigma=\left(\sigma_{i j}\right)$ is the stress tensor defined by $\sigma_{i j}(x)=\sum_{k l} C_{i j k l}(x) \partial_{l} u_{k}$ and $n$ is the unit outer normal of $\partial \Omega$.

In order to motivate our study, let us briefly discuss the isotropic case. Here the existence and uniqueness of the radiation solution to the exterior boundary value problems have been established in [2] and [10, Chapter 11]. The uniqueness is assured by imposing appropriate radiation conditions at infinity, which are called Sommerfeld-Kupradze radiation conditions (see 9]). The existence is shown by the limiting absorption principle, which is proved by a general approach initiated by Eidus [5]. Note that the results in [2] and [10] are valid for two or three dimensions. Besides the radiation conditions, one of the key ingredients in 2 and [10] is the unique continuation property for the isotropic elasticity system. The unique continuation property for this system has been proved by several authors; see for example [1], [3], [17, and [18. In contrast to the isotropic setup, the unique continuation property for the general anisotropic elasticity system still poses a challenging open problem. Recently, the authors succeeded in proving the unique continuation property for the two-dimensional anisotropic system under some generic assumptions, which paves the way for establishing the limiting absorption principle and which enables them to prove existence and uniqueness of the exterior boundary value problem for the same system. The study of this work is motivated by related inverse problems in the anisotropic elasticity system. The existence and uniqueness of the radiation solution are fundamental to the investigation of inverse problems. 
Although we assume that the elastic tensor $C(x)$ is homogeneous for large $|x|$, it still preserves the anisotropic character. Therefore, the classical SommerfeldKupradze radiation conditions are not applicable in our case. In order to derive suitable radiation conditions for the anisotropic system, we are led to analyze the radiation pattern of the fundamental solution for $L(D, \omega):=\nabla \cdot(C \nabla \bullet)+\omega^{2} I=$ $L(D)+\omega^{2} I$. This is bound to be a complicated problem due to anisotropy. Nevertheless, when the slowness curves of the homogeneous system $L(D, \omega) u=0$ satisfy certain "good" conditions (see (2.3), (2.4) ), one can still analyze the radiation pattern of the fundamental solution and derive appropriate radiation conditions. This was done by Natroshvili in [14. In fact, before Natroshvili's work, Wilcox studied the steady-state wave propagation problem in a class of anisotropic media governed by first order systems of partial differential equations and derived similar radiation conditions 20]. Working in the same first order systems, Schulenberger and Wilcox also investigated the Rellich-type uniqueness theorem and the limiting absorption principle when the medium is inhomogeneous [15, [16]. Perhaps the article [16] is most closely related to the considerations of the present paper. Although the elastic wave equations in an inhomogeneous anisotropic medium can be put into a first order symmetric hyperbolic system [19, it is more natural to consider the original second order system when we are dealing with the exterior boundary value problem for the steady-state equations. On the other hand, the results in [16] (also [15]) were proved by avoiding the point spectrum of the associated inhomogeneous anisotropic operator due to the lack of the unique continuation property.

This paper is organized as follows. In Section 2, we recall some of Natroshvili's results that are needed later on. We also use this opportunity to briefly review the unique continuation property proved by the authors. In Section 3, we formally define the radiation solutions to the exterior Dirichlet and Neumann boundary value problems and establish the uniqueness theorem. The limiting absorption principle is then proved by means of several lemmas in Section 4. In Section 5 we discuss the spectra of the exterior Dirichlet and Neumann operators.

\section{Preliminaries}

2.1. Outgoing (incoming) fundamental solution. For the sake of completeness, we give a brief review of Natroshvili's results in [14 about the outgoing (incoming) fundamental solution for the homogeneous system. Similar results can also be found in [12] and 20] for first order systems. Let us consider a homogeneous elastic tensor $C$ satisfying (1.2) and (1.3). Denote $\Gamma(x, \omega)$ the fundamental solution of $L(D, \omega)$, i.e.,

$$
L(D, \omega) \Gamma(x, \omega)=\left(L(D)+\omega^{2} I\right) \Gamma(x, \omega)=\delta(x) I,
$$

where $L(D)$ is a matrix differential operator with components given by

$$
L_{i k}(D)=\sum_{j l} C_{i j k l} \partial_{j} \partial_{l} .
$$

Taking the Fourier transform of (2.1), we obtain that

$$
\left(\omega^{2} I-L(\xi)\right) \hat{\Gamma}(\xi, \omega)=I .
$$


Here the $2 \times 2$ matrix $L(\xi)$ is described by

$$
L_{i k}(\xi)=\sum_{j l} C_{i j k l} \xi_{j} \xi_{l} .
$$

We now denote $L_{11}(\xi)=\alpha(\xi), L_{12}(\xi)=L_{21}(\xi)=\gamma(\xi)$, and $L_{22}(\xi)=\beta(\xi)$. From (1.3), it is clear that $\alpha(\xi)>0$ and $\beta(\xi)>0$ for all $\xi \neq 0$. Define

$$
\phi(\xi, \omega):=\operatorname{det}\left(\omega^{2} I-L(\xi)\right)=\alpha(\xi) \beta(\xi)-\gamma^{2}(\xi)-\omega^{2}[\alpha(\xi)+\beta(\xi)]+\omega^{4} .
$$

Introducing the polar coordinates

$$
\xi_{1}=\rho \cos \theta, \xi_{2}=\rho \sin \theta,
$$

we get from (2.2) that

$$
\begin{aligned}
\phi(\xi, \omega) & =\rho^{4} \alpha(\eta) \beta(\eta)-\rho^{4} \gamma^{2}(\eta)-\omega^{2}[\alpha(\eta)+\beta(\eta)] \rho^{2}+\omega^{4} \\
& =\phi(\eta)\left\{\rho^{4}-\phi^{-1}(\eta) \omega^{2}[\alpha(\eta)+\beta(\eta)] \rho^{2}+\phi^{-1}(\eta) \omega^{4}\right\} \\
& =\phi(\eta)\left(\rho^{2}-\omega^{2} k_{1}^{2}(\theta)\right)\left(\rho^{2}-\omega^{2} k_{2}^{2}(\theta)\right),
\end{aligned}
$$

where $\eta=\eta(\theta)=(\cos \theta, \sin \theta), \phi(\eta)=\operatorname{det}\left(\alpha(\eta) \beta(\eta)-\gamma^{2}(\eta)\right)>0$, and

$$
k_{j}^{2}(\theta)=(2 \phi(\eta))^{-1}\left\{(\alpha(\eta)+\beta(\eta))+(-1)^{j} \sqrt{(\alpha(\eta)-\beta(\eta))^{2}+4 \gamma^{2}(\eta)}\right\}>0 .
$$

In the following, we will also denote $\rho_{j}=\omega k_{j}$. Let the curve $S_{j}$ be defined by $\left\{(\rho, \theta): \rho=\omega k_{j}(\theta), 0 \leq \theta \leq 2 \pi\right\}$. Obviously, $\phi(\xi, \omega)$ vanishes on the curve $S_{j}$. We now assume that

$$
k_{1}(\theta)<k_{2}(\theta) \quad \forall 0 \leq \theta \leq 2 \pi
$$

and

$$
S_{2} \text { is a strictly convex curve. }
$$

Notice that the convexity of $S_{1}$ is also assumed in [14. However, this assumption is redundant. The convexity of $S_{1}$ is a well-known property in the theory of anisotropic elastic waves (see 4 for detailed arguments). From (2.3) we can see that $\nabla \phi(\xi, \omega) \neq$ 0 on $S_{j}$ for all $j$. Furthermore, it follows from (2.4) that for any $x \neq 0$ there exists a unique point $\xi^{j}$ on $S_{j}$ such that the unit outer normal vector $\tilde{n}\left(\xi^{j}\right)$ of $S_{j}$ at $\xi^{j}$ is parallel to $x$, denoted by $\tilde{n}\left(\xi^{j}\right) \| x$. Since $\phi(-\xi, \omega)=\phi(\xi, \omega)$, the normal vector $\tilde{n}\left(-\xi^{j}\right)$ is equal to $-\tilde{n}\left(\xi^{j}\right)$. In fact, in terms of $\phi(\xi, \omega)$, the unit normal vector $\tilde{n}\left(\xi_{j}\right)$ is given by

$$
\tilde{n}\left(\xi_{j}\right)=(-1)^{j} \frac{\nabla \phi\left(\xi_{j}, \omega\right)}{\left|\nabla \phi\left(\xi_{j}, \omega\right)\right|} .
$$

Moreover, it is readily seen that there exists positive constants $\delta_{1}$ and $\delta_{2}$ so that

$$
\delta_{1} \leq k_{1}(\theta)<k_{2}(\theta) \leq \delta_{2} \quad \forall 0 \leq \theta \leq 2 \pi .
$$

Based on conditions (2.3) and (2.4), Natroshvili constructed fundamental solutions for $L(D, \omega)$ by considering the limits of $\Gamma\left(x, \tau_{\varepsilon}\right)$ as $\pm \varepsilon \rightarrow 0$, where $\tau_{\varepsilon}=\omega+\imath \varepsilon$, and

$$
L\left(D, \tau_{\varepsilon}\right) \Gamma\left(x, \tau_{\varepsilon}\right)=\left(L(D)+\tau_{\varepsilon}^{2} I\right) \Gamma\left(x, \tau_{\varepsilon}\right)=\delta(x) I .
$$

In fact, by the light of Fourier transform, $\Gamma\left(x, \tau_{\varepsilon}\right)$ is given by

$$
\Gamma\left(x, \tau_{\varepsilon}\right)=\frac{1}{4 \pi^{2}} \int_{\mathbb{R}^{2}} \phi^{-1}\left(\xi, \tau_{\varepsilon}\right) L^{*}\left(\xi, \tau_{\varepsilon}\right) e^{\imath x \xi} d \xi,
$$


where $L^{*}\left(\xi, \tau_{\varepsilon}\right)$ is the adjoint of the matrix $\tau_{\varepsilon}^{2} I-L(\xi)$, i.e.,

$$
L^{*}\left(\xi, \tau_{\varepsilon}\right)=\left[\begin{array}{cc}
\tau_{\varepsilon}^{2}-\beta(\xi) & \gamma(\xi) \\
\gamma(\xi) & \tau_{\varepsilon}^{2}-\alpha(\xi)
\end{array}\right] .
$$

Here $\phi\left(\xi, \tau_{\varepsilon}\right)$ is defined as in (2.2) with $\omega$ being replaced by $\tau_{\varepsilon}$. It is easy to see that the matrix $\Gamma\left(x, \tau_{\varepsilon}\right) \in C^{\infty}\left(\mathbb{R}^{2} \backslash\{0\}\right)$ and, together with all its derivatives, decay exponentially in $|x|$ as $|x| \rightarrow \infty$. We now summarize Natroshvili's results in the following theorem.

Theorem 2.1 ([14, Theorem 7 \& Corollary 8]). Assume that (2.3) and (2.4) hold. Then

(i) the limits

$$
\lim _{ \pm \varepsilon \rightarrow 0} \Gamma\left(x, \tau_{\varepsilon}\right)=\Gamma_{ \pm}(x, \omega)
$$

exist for all $x \neq 0$ and uniformly in $|x|>a>0$;

(ii) $\Gamma_{ \pm}(x, \omega)$ are fundamental solutions of $L(D, \omega)$ defined by

$$
\begin{aligned}
\Gamma_{ \pm}(x, \omega)= & (2 \pi)^{-2} \int_{\mathbb{R}^{2}}[1-h(\xi)]\left[\omega^{2} I-L(\xi)\right]^{-1} e^{\imath x \xi} d \xi \\
& +(2 \pi)^{-2} \text { p.v. } \int_{\mathbb{R}^{2}} h(\xi)\left[\omega^{2} I-L(\xi)\right]^{-1} e^{\imath x \xi} d \xi \\
& \pm(4 \pi)^{-1} \imath \sum_{j} \int_{S_{j}}(-1)^{j} L^{*}(\xi, \omega)|\nabla \phi(\xi, \omega)|^{-1} e^{\imath x \xi} d S_{j},
\end{aligned}
$$

where $h \in C^{\infty}\left(\mathbb{R}^{2}\right)$ is given by

$$
h(\xi)=\left\{\begin{array}{lll}
1 & \text { for } & |\xi|<c_{0}, \\
0 & \text { for } & |\xi|>2 c_{0}
\end{array}\right.
$$

with $c_{0}>2 \delta_{2} \omega$, p.v. stands for the principal value, $L^{*}(\xi, \omega)$ is the adjoint of the matrix $\omega^{2} I-L(\xi)$ defined similarly as above, $d S_{j}$ is the arc-length element of $S_{j}$, and moreover,

$$
\Gamma_{ \pm}(x, \omega)=\Gamma_{ \pm}(-x, \omega)=\Gamma_{ \pm}(x, \omega)^{t} ;
$$

(iii) in the vicinity of the origin $(0<|x|<1 / 2)$ the following estimates hold:

$$
\left|\partial^{\nu}\left(\Gamma_{ \pm}(x, \omega)-\Gamma(x)\right)\right|<c r_{|\nu|}(x), \quad|\nu|=0,1,2,3,
$$

where $r_{0}(x)=r_{1}(x)=1, r_{2}(x)=\ln |x|^{-1}, r_{3}(x)=|x|^{-1}$, and $\Gamma(x)$ is the fundamental solution of $L(D)$, i.e.,

$$
L(D) \Gamma(x)=\delta(x) I
$$

(vi) for sufficiently large $|x|$, we have the following asymptotic behaviors:

$$
\begin{aligned}
& \Gamma_{+}(x, \omega)=\sum_{j}|x|^{-1 / 2} R_{+, j} e^{\imath x \xi^{j}}+O\left(|x|^{-2 / 3}\right), \\
& \Gamma_{-}(x, \omega)=\sum_{j}|x|^{-1 / 2} R_{-, j} e^{-\imath x \xi^{j}}+O\left(|x|^{-2 / 3}\right),
\end{aligned}
$$

where $\xi^{j} \in S_{j}$ with $\tilde{n}\left(\xi^{j}\right)$ parallel to $x$ and

$$
R_{+, j}(x)=\overline{R_{-, j}}(x)=(-1)^{j} \frac{\imath}{\sqrt{2 \pi}} \frac{e^{\imath \pi / 4}}{\sqrt{\aleph\left(\xi^{j}\right)}\left|\nabla \phi\left(\xi^{j}, \omega\right)\right|} L^{*}\left(\xi^{j}, \omega\right),
$$

where $\aleph\left(\xi^{j}\right)$ is the curvature of $S_{j}$ at $\xi^{j}$; 
(v) if $y$ is in any bounded subset of $\mathbb{R}^{2}$, then we have that for any multi-indices $\alpha, \beta$

$$
\partial_{x}^{\alpha} \partial_{y}^{\beta} \Gamma_{ \pm}(x-y, \omega)=\sum_{j}|x|^{-1 / 2} R_{ \pm, j}(x)\left( \pm \xi^{j}\right)^{\alpha}\left(\mp \xi^{j}\right)^{\beta} e^{ \pm \imath(x-y) \xi^{j}}+O\left(|x|^{-3 / 2}\right)
$$

as $|x| \rightarrow \infty$.

Remark 2.2. $\Gamma_{+}(x, \omega)$ corresponds to the outgoing fundamental solution and $\Gamma_{-}(x, \omega)$ is the incoming fundamental solution.

Remark 2.3. In $\mathbb{R}^{2}$, the explicit form of $\Gamma(x)$ is given by

$$
\Gamma(x)=\frac{1}{(2 \pi)^{2}} \int_{\eta \in \mathbb{S}^{1}} \ln |\eta \cdot x| L^{-1}(\eta) d \eta
$$

(see [6, Page 127]). It is easy to see that $\Gamma(x)=O(\ln |x|)$ near the origin.

Now we are ready to define the radiation conditions for the anisotropic elasticity system $L(D, \omega)$. Let $u(x)$ be defined in $\Omega$ and $C^{1}$ for large $|x|$. Then $u(x)$ is said to satisfy the generalized Sommerfeld-Kupradze outgoing (incoming) radiation conditions if

$$
\left\{\begin{array}{l}
u(x)=\sum_{j} u^{(j)}(x), u^{(j)}=O\left(|x|^{-1 / 2}\right), \\
\partial_{l} u^{(j)}(x)-\imath \xi_{l}^{j} u^{(j)}(x)=O\left(|x|^{-3 / 2}\right), j, l=1,2,
\end{array}\right.
$$

hold, where $\xi^{j}=\left[\xi_{1}^{j}, \xi_{2}^{j}\right]^{t}$ is the vector on $S_{j}$ with $\tilde{n}\left(\xi^{j}\right) \| x$. When $u(x)$ is a vector or a matrix, we say that $u(x)$ satisfies (2.5) if each component of $u$ satisfies (2.5). It is clear that $\Gamma_{+}(x, \omega)\left(\Gamma_{-}(x, \omega)\right)$ satisfies the outgoing (incoming) radiation conditions (2.5). Similar to the isotropic case, if $u$ satisfies the radiation conditions (2.5), then it has an integral representation. More precisely, let $u \in C^{1}(\bar{\Omega}) \cap H^{2}(\Omega)$ satisfy the radiation conditions (2.5) and $L(D, \omega) u$ be compactly supported. Then

$$
\begin{aligned}
u(x)= & \int_{\Omega} \Gamma_{ \pm}(x-y, \omega) L\left(D_{y}, \omega\right) u(y) d y+\int_{\partial \Omega}\left\{\Gamma_{ \pm}(x-y, \omega)\left[T\left(D_{y}, n(y)\right) u(y)\right]\right. \\
& \left.-\left[T\left(D_{y}, n(y)\right) \Gamma_{ \pm}(x-y, \omega)\right]^{t} u(y)\right\} d S \quad \forall x \in \Omega
\end{aligned}
$$

(see [14]).

2.2. Unique continuation property. Here we would like to state a recent unique continuation result for the two-dimensional anisotropic elasticity system established in 13 . Assume that $\Omega_{0}$ is any bounded open connected domain in $\mathbb{R}^{2}$. As before, we suppose that the elastic tensor $C(x)$ satisfies the symmetry properties (1.2). Instead of the strong convexity condition (1.3), to guarantee the unique continuation property, it suffices to impose the strong ellipticity condition on $C(x)$, namely, there exists $\tilde{\delta}>0$ so that for any vectors $a=\left[a_{1}, a_{2}\right]^{t}$ and $b=\left[b_{1}, b_{2}\right]^{t}$ we have that

$$
C_{i j k l}(x) a_{i} b_{j} a_{k} b_{l} \geq \tilde{\delta}|a|^{2}|b|^{2}
$$

for all $x \in \Omega_{0}$. It is obvious that (1.3) implies (2.7). For the regularity of $C(x)$, we assume that each component of $C(x)$ is Lipschitz in $\Omega_{0}$. Denote

$$
\Lambda_{11}=\left(C_{i 1 k 1}\right), \Lambda_{22}=\left(C_{i 2 k 2}\right), \Lambda_{12}=\Phi+\Phi^{t} \text { with } \Phi=\left(C_{i 2 k 1}\right) .
$$

The following unique continuation result is proved in [13]. 
Theorem 2.4. Let $(a(x), z(x))$ be an eigenpair of the quadratic matrix polynomial

$$
\Lambda_{11} p^{2}+\Lambda_{12} p+\Lambda_{22},
$$

i.e.

$$
\left(\Lambda_{11} a^{2}+\Lambda_{12} a+\Lambda_{22}\right) z=0 \quad \forall x \in \Omega_{0} .
$$

Assume that $a(x), z(x)$ are Lipschitz and the matrix $[z, \bar{z}]$ is nonsingular for all $x \in \Omega_{0}$. Then the operator $\nabla \cdot(C(x) \nabla \bullet)+\omega^{2} I$ possesses the unique continuation property. In other words, if $u \in H_{\text {loc }}^{2}\left(\Omega_{0}\right)$ satisfies $\nabla \cdot(C(x) \nabla u)+\omega^{2} u=0$ in $\Omega_{0}$ and $u=0$ in any non-empty open subset of $\Omega_{0}$, then $u \equiv 0$ in $\Omega_{0}$.

The proof of Theorem 2.4 relies on converting the two-dimensional elasticity system $\nabla \cdot(C(x) \nabla u)+\omega^{2} u=0$ into a first-order elliptic system and using suitable Carleman-type estimates.

\section{EXTERIOR BOUNDARY VALUE PROBLEMS}

In this section we would like to discuss the exterior boundary value problem for the system (1.1) with Dirichlet condition (1.4) or Neumann condition (1.5) in more detail. Before doing so, we want to precisely state what conditions we will impose on the elastic tensor $C(x)$. We assume that $C(x)$ is a Lipschitz function in $\Omega$ satisfying the symmetry properties (1.2), the strong convexity condition (1.3), and $C(x)=C$ for all $|x| \geq R>0$ for some constant anisotropic elastic tensor $C$. Furthermore, we suppose that the assumptions in Theorem 2.4 hold for $C(x)$ in $\Omega_{2 R}:=\Omega \cap\{|x|<2 R\}$, which guarantee the validity of the unique continuation property in $\Omega_{2 R}$. In this section and the remaining sections, we always assume that $C(x)$ satisfies the above conditions.

In order to put the boundary value problem in the weak formulation, we define two operators.

Definition 3.1. Let the bilinear form $B(\cdot, \cdot)$ be defined by

$$
\begin{aligned}
B(u, v) & =\int_{\Omega} \sum_{i j k l} C_{i j k l}(x) \partial_{l} u_{k} \overline{\partial_{j} v_{i}} d x=\int_{\Omega} \sum_{i j} \sigma_{i j} \overline{\partial_{j} v_{i}} d x \\
& =\int_{\Omega} C(x) \epsilon(u) \cdot \overline{\epsilon(v)} d x, \quad \forall u, v \in H^{1}(\Omega),
\end{aligned}
$$

where $(\epsilon(u))_{i j}=(1 / 2)\left(\partial_{j} u_{i}+\partial_{i} u_{j}\right)$.

(i) The operator for the Dirichlet boundary value problem is given by $\mathcal{L}^{D}$ : $L^{2}(\Omega) \rightarrow L^{2}(\Omega)$ with the domain

$$
\mathcal{D}\left(\mathcal{L}^{D}\right)=\left\{u \in H_{0}^{1}(\Omega): \exists h \in L^{2}(\Omega), \forall v \in H_{0}^{1}(\Omega), B(u, v)=(h, v)\right\}
$$

and $\mathcal{L}^{D} u=-\nabla \cdot(C(x) \nabla u)$ for $u \in C_{0}^{\infty}(\Omega)$.

(ii) The operator for the Neumann boundary value problem is given by $\mathcal{L}^{N}$ : $L^{2}(\Omega) \rightarrow L^{2}(\Omega)$ with the domain

$$
\mathcal{D}\left(\mathcal{L}^{N}\right)=\left\{u \in H^{1}(\Omega): \exists h \in L^{2}(\Omega), \forall v \in H^{1}(\Omega), B(u, v)=(h, v)\right\}
$$

and $\mathcal{L}^{N} u=-\nabla \cdot(C(x) \nabla u)$ for $u \in C^{\infty}(\Omega)$.

It is not hard to see that $\mathcal{L}^{D}$ and $\mathcal{L}^{N}$ are symmetric and self-adjoint. Also, it follows from Korn's inequalities that the bilinear form is coercive in $H_{0}^{1}(\Omega)$ and $H^{1}(\Omega)$. 
To formulate the exterior boundary value problem with radiation conditions, we introduce two weighted Sobolev spaces. Let $\varrho=(1+|x|)^{-s}$ with $s>1 / 2$ and the weighted inner product

$$
(f, g)_{H^{1,-s}(\Omega)}=(\varrho f, \varrho g)+(\varrho \nabla f, \varrho \nabla g) \text { for } f, g \in H_{\mathrm{loc}}^{1}(\Omega) .
$$

Denote

$$
H_{0}^{1,-s}(\Omega)=\text { completion of } H_{0}^{1}(\Omega) \text { and } \quad H^{1,-s}(\Omega)=\text { completion of } H^{1}(\Omega)
$$

with completion being defined in terms of $\|f\|_{H^{1,-s}(\Omega)}=(f, f)_{H^{1,-s}(\Omega)}^{1 / 2}$.

Definition 3.2. (i) A function $u \in H_{0}^{1,-s}(\Omega)$ which is $C^{1}$ smooth for sufficiently large $|x|$ is called an outgoing (incoming) radiation solution of the Dirichlet problem

$$
\mathcal{L}^{D} u-\omega^{2} u=f
$$

if and only if $\forall \psi(x) \in C_{0}^{\infty}(\Omega), B(u, \psi)-\omega^{2}(u, \psi)=(f, \psi)$ and $u$ satisfies the outgoing (incoming) radiation conditions (2.5).

(ii) A function $u \in H^{1,-s}(\Omega)$ which is $C^{1}$ smooth for sufficiently large $|x|$ is called an outgoing (incoming) radiation solution of the Neumann problem

$$
\mathcal{L}^{N} u-\omega^{2} u=f
$$

if and only if $\forall \psi(x) \in C^{\infty}(\Omega) \cap C_{0}^{\infty}\left(\mathbb{R}^{2}\right), B(u, \psi)-\omega^{2}(u, \psi)=(f, \psi)$ and $u$ satisfies the outgoing (incoming) radiation conditions (2.5).

Our first task is to show that the radiation solution is unique. For definiteness, we consider the case of outgoing radiation solution for the Dirichlet problem. The same method works for other types of solutions. To begin, we prove that a radiation solution with homogeneous data decays at a rate $O\left(|x|^{-3 / 2}\right)$ at infinity.

Lemma 3.3. Let $u(x)$ be an outgoing radiation solution of the homogeneous Dirichlet problem, i.e. $f=0$. Then $u(x)=O\left(|x|^{-3 / 2}\right)$ as $|x| \rightarrow \infty$.

Proof. The proof of this lemma is inspired by the arguments in 14. Since $C(x)=C$ for $|x|>R$, using the representation formula (2.6) in $\left\{|x|>R^{\prime}\right\}$ with $R^{\prime}>R$ and taking into account of $f=0$, we obtain that

$$
u(x)=\int_{\Sigma_{R^{\prime}}}\left\{\Gamma_{+}(x-y, \omega)\left[T\left(D_{y}, \eta(y)\right) u(y)\right]-\left[T\left(D_{y}, \eta(y)\right) \Gamma_{+}(x-y, \omega)\right]^{t} u(y)\right\} d S
$$

for all $|x|>R^{\prime}$, where $\Sigma_{R^{\prime}}=\left\{|x|=R^{\prime}\right\}$ and $\eta(y)=y /|y|$. By the asymptotic behavior of $\Gamma_{+}(x-y, \omega)$ (see (v) of Theorem 2.1), we can see that

$$
\Gamma_{+}(x-y, \omega)=\sum_{j}|x|^{-1 / 2} L^{*}\left(\xi^{j}, \omega\right) a_{j}\left(\xi^{j}, y\right) e^{\imath x \xi^{j}}+O\left(|x|^{-3 / 2}\right)
$$

and

$$
\begin{aligned}
& {\left[T\left(D_{y}, \eta(y)\right) \Gamma_{+}(x-y, \omega)\right]^{t}=-\left[T\left(D_{x}, \eta(y)\right) \Gamma_{+}(x-y, \omega)\right]^{t}} \\
& \quad=-\sum_{j}|x|^{-1 / 2} L^{*}\left(\xi^{j}, \omega\right)\left[T\left(\imath \xi^{j}, \eta(y)\right)\right]^{t} a_{j}\left(\xi^{j}, y\right) e^{i x \xi^{j}}+O\left(|x|^{-3 / 2}\right),
\end{aligned}
$$

where $a_{j}\left(\xi^{j}, y\right)$ is a scalar function. Substituting (3.2) and (3.3) into the representation formula (3.1), we get that

$$
u(x)=\sum_{j} u^{(j)}=\sum_{j}|x|^{-1 / 2} L^{*}\left(\xi^{j}, \omega\right) H_{j}\left(\xi^{j}\right) e^{\imath x \xi^{j}}+O\left(|x|^{-3 / 2}\right),
$$


where $H_{j}=\left[h_{j 1}, h_{j 2}\right]^{t}$ is determined by $u$ on $\Sigma_{R^{\prime}}$. Clearly $u=\sum_{j} u^{(j)}$ satisfies the outgoing radiation conditions (2.5). Similarly, we can derive that

$$
\begin{aligned}
T(D, \nu) u(x) & =T(D, \nu) \sum_{j} u^{(j)} \\
& =\imath \sum_{j}|x|^{-1 / 2} T\left(\xi^{j}, \nu\right) L^{*}\left(\xi^{j}, \omega\right) H_{j}\left(\xi^{j}\right) e^{\imath x \xi^{j}}+O\left(|x|^{-3 / 2}\right)
\end{aligned}
$$

for any vector $\nu(x)$.

Now letting $\tilde{R}>R$ and using the Green formula in $\Omega_{\tilde{R}}$, we obtain that

$$
\begin{aligned}
& \int_{\Sigma_{\tilde{R}}}\{T(D, \eta(x)) u(x) \cdot \overline{u(x)}-u(x) \cdot \overline{T(D, \eta(x)) u(x)}\} d S \\
& \quad=\int_{\partial \Omega}\{T(D, n) u(x) \cdot \overline{u(x)}-u(x) \cdot \overline{T(D, n) u(x)}\} d S=0
\end{aligned}
$$

since $u(x)=0$ on $\partial \Omega$, where $n$, as defined before, is the unit outer normal of $\partial \Omega$. Here we want to remark that the traction operator $T$ is defined in terms of the inhomogeneous elastic tensor $C(x)$, which is homogeneous on $\Sigma_{\tilde{R}}$ for all $\tilde{R}>R$. It follows from (3.6) that for sufficiently large $\ell$

$$
\frac{1}{\ell} \int_{\ell}^{2 \ell} \int_{\Sigma_{\tilde{R}}} \sum_{k, j}\left\{T(D, \eta) u^{(k)}(x) \cdot \overline{u^{(j)}(x)}-u^{(j)}(x) \cdot \overline{T(D, \eta) u^{(k)}(x)}\right\} d S d \tilde{R}=0 .
$$

We first deal with the integrands in (3.7) with $k \neq j$. It is enough to consider the term $T(D, \eta) u^{(1)} \cdot \overline{u^{(2)}}$. By the light of the radiation conditions (2.5), we can deduce that

$$
\begin{aligned}
\frac{1}{\ell} & \int_{\ell}^{2 \ell} \int_{\Sigma_{\tilde{R}}} T(D, \eta) u^{(1)}(x) \cdot \overline{u^{(2)}(x)} d S d \tilde{R} \\
& =\frac{1}{\ell} \int_{\ell}^{2 \ell} \int_{\Sigma_{\tilde{R}}} \sum_{i j k l} C_{i j k l} \partial_{l} u_{k}^{(1)}(x) \overline{\eta_{j}} \overline{u_{i}^{(2)}(x)} d S d \tilde{R} \\
& =\frac{1}{\ell} \int_{\ell}^{2 \ell} \int_{\Sigma_{\tilde{R}}} \sum_{i j k l} C_{i j k l} \eta_{j}\left\{\left(\imath \xi_{l}^{1}\right) u_{k}^{(1)}(x)+O\left(|x|^{-3 / 2}\right)\right\} \overline{u_{i}^{(2)}(x)} d S d \tilde{R} \\
& =\frac{1}{\ell} \int_{\ell}^{2 \ell} \int_{\Sigma_{\tilde{R}}}\left\{\sum_{i k} A_{i k}(\eta) u_{k}^{(1)}(x) \overline{u_{i}^{(2)}(x)}+O\left(|x|^{-2}\right)\right\} d S d \tilde{R} \\
& =\frac{1}{\ell} \int_{\ell}^{2 \ell} \int_{\Sigma_{\tilde{R}}} \sum_{i k} A_{i k}(\eta) u_{k}^{(1)}(\tilde{R} \eta) \overline{u_{i}^{(2)}(\tilde{R} \eta)} d S d \tilde{R}+O\left(\ell^{-1}\right),
\end{aligned}
$$

where $A_{i k}=\sum_{j l} C_{i j k l} \eta_{j}\left(\imath \xi_{l}^{1}\right)$. Again, in view of the radiation conditions, we have that

$$
\begin{array}{ll}
u_{k}^{(1)}(\tilde{R} \eta)=O\left(\tilde{R}^{-1 / 2}\right), & \frac{\partial}{\partial \tilde{\tilde{R}}} u_{k}^{(1)}(\tilde{R} \eta)-\imath \vartheta^{1}(\eta) u_{k}^{(1)}=O\left(\tilde{R}^{-3 / 2}\right), \\
u_{i}^{(2)}(\tilde{R} \eta)=O\left(\tilde{R}^{-1 / 2}\right), & \frac{\partial}{\partial \tilde{R}} u_{i}^{(2)}(\tilde{R} \eta)-\imath \vartheta^{2}(\eta) u_{i}^{(2)}=O\left(\tilde{R}^{-3 / 2}\right),
\end{array}
$$

where $\vartheta^{j}(\eta)=\xi^{j} \cdot \eta$. Recall that here $\xi^{j}$ is the point on $S_{j}$ related to $\eta$. Since $S_{j}$ is strictly convex for each $j$, we can see that

$$
\vartheta^{j}(\eta)>0 \text { and } \vartheta^{1}(\eta)-\vartheta^{2}(\eta)=\left(\xi^{1}-\xi^{2}, \eta\right) \neq 0 \text { for all } \eta \text {. }
$$


We now compute

$$
\begin{aligned}
& \frac{1}{\ell} \int_{\ell}^{2 \ell} \int_{\Sigma_{\tilde{R}}} \sum_{i k} A_{i k}(\eta) u_{k}^{(1)}(\tilde{R} \eta) \overline{u_{i}^{(2)}(\tilde{R} \eta)} d S d \tilde{R} \\
& =\frac{1}{\ell} \int_{\ell}^{2 \ell} \int_{\Sigma_{\tilde{R}}} \sum_{i k} \frac{(-\imath) A_{i k}(\eta)}{\vartheta^{1}(\eta)-\vartheta^{2}(\eta)}\left[\imath \vartheta^{1} u_{k}^{(1)}\left(\tilde{R} \eta \overline{u_{i}^{(2)}(\tilde{R} \eta)}+u_{k}^{(1)}(\tilde{R} \eta) \overline{\imath \vartheta^{2} u_{i}^{(2)}(\tilde{R} \eta)}\right] d S d \tilde{R}\right. \\
& =\frac{1}{\ell} \int_{\ell}^{2 \ell} \int_{\Sigma_{\tilde{R}}} \sum_{i k} \frac{(-\imath) A_{i k}(\eta)}{\vartheta^{1}(\eta)-\vartheta^{2}(\eta)}\left[\frac{\partial}{\partial \tilde{R}} u_{k}^{(1)}(\tilde{R} \eta) \overline{u_{i}^{(2)}(\tilde{R} \eta)}+u_{k}^{(1)}(\tilde{R} \eta) \frac{\partial}{\partial \tilde{R}} \overline{u_{i}^{(2)}(\tilde{R} \eta)}\right] d S d \tilde{R} \\
& \quad+O\left(\ell^{-1}\right) \\
& =\frac{1}{\ell} \int_{0}^{2 \pi} \sum_{i k} \frac{(-\imath) A_{i k}(\eta)}{\vartheta^{1}(\eta)-\vartheta^{2}(\eta)} \int_{\ell}^{2 \ell} \frac{\partial}{\partial \tilde{R}}\left[u_{k}^{(1)}(\tilde{R} \eta) \overline{u_{i}^{(2)}(\tilde{R} \eta)}\right] \tilde{R} d \tilde{R} d \theta \\
& \quad+O\left(\ell^{-1}\right) \\
& =\frac{1}{\ell} \int_{0}^{2 \pi} \sum_{i k} \frac{(-\imath) A_{i k}(\eta)}{\vartheta^{1}(\eta)-\vartheta^{2}(\eta)}\left\{\left[\tilde{R} u_{k}^{(1)}(\tilde{R} \eta) \overline{u_{i}^{(2)}(\tilde{R} \eta)}\right]_{\ell}^{2 \ell}-\int_{\ell}^{2 \ell} u_{k}^{(1)}(\tilde{R} \eta) \overline{u_{i}^{(2)}(\tilde{R} \eta)} d \tilde{R}\right\} d \theta \\
& \quad+O\left(\ell^{-1}\right) \\
& =O\left(\ell^{-1}\right),
\end{aligned}
$$

where in the third equality we have set $\eta(\theta)=(\cos \theta, \sin \theta)$. Combining (3.8) and (3.9) leads to

$$
\frac{1}{\ell} \int_{\ell}^{2 \ell} \int_{\Sigma_{\tilde{R}}} T(D, \eta) u^{(1)}(x) \cdot \overline{u^{(2)}(x)} d S d \tilde{R}=O\left(\ell^{-1}\right)
$$

and hence

$$
\frac{1}{\ell} \int_{\ell}^{2 \ell} \int_{\Sigma_{\tilde{R}}} T(D, \eta) u^{(k)}(x) \cdot \overline{u^{(j)}(x)} d S d \tilde{R}=O\left(\ell^{-1}\right)
$$

for all $k \neq j$. Plugging (3.4), (3.5) into (3.7) and using (3.10), we obtain that

$$
\begin{aligned}
& \frac{1}{\ell} \int_{\ell}^{2 \ell} \int_{\Sigma_{\tilde{R}}} \sum_{j}|x|^{-1}\left[T\left(\xi^{j}, \eta\right) L^{*}\left(\xi^{j}, \omega\right) H_{j}\left(\xi^{j}\right) \cdot L^{*}\left(\xi^{j}, \omega\right) \overline{H_{j}\left(\xi^{j}\right)}\right. \\
& \left.\quad+T\left(\xi^{j}, \eta\right) L^{*}\left(\xi^{j}, \omega\right) \overline{H_{j}\left(\xi^{j}\right)} \cdot L^{*}\left(\xi^{j}, \omega\right) H_{j}\left(\xi^{j}\right)\right] d S d \tilde{R}=O\left(\ell^{-1}\right) .
\end{aligned}
$$

Letting $\ell \rightarrow \infty$ in (3.11) gives

$$
\begin{aligned}
& \sum_{j} \int_{0}^{2 \pi} T\left(\xi^{j}, \eta\right) L^{*}\left(\xi^{j}, \omega\right) H_{j}\left(\xi^{j}\right) \cdot L^{*}\left(\xi^{j}, \omega\right) \overline{H_{j}\left(\xi^{j}\right)} \\
& \quad+T\left(\xi^{j}, \eta\right) L^{*}\left(\xi^{j}, \omega\right) \overline{H_{j}\left(\xi^{j}\right)} \cdot L^{*}\left(\xi^{j}, \omega\right) H_{j}\left(\xi^{j}\right) d \theta=0 .
\end{aligned}
$$

Now it is useful to take a closer look at $L^{*}\left(\xi^{j}, \omega\right)$. Since $\operatorname{det}\left(L^{*}\left(\xi^{j}, \omega\right)\right)=$ $\phi\left(\xi^{j}, \omega\right)=0$, the two column vectors of $L^{*}\left(\xi^{j}, \omega\right)$ must be linearly dependent. More precisely, let $W^{1}(\xi, \omega):=\left[\omega^{2}-\beta(\xi), \gamma(\xi)\right]^{t}$ and $W^{2}(\xi, \omega):=\left[\gamma(\xi), \omega^{2}-\alpha(\xi)\right]^{t}$ be two column vectors of $L^{*}(\xi, \omega)$. Then there exists a scalar function $c(\xi)$ such that

$$
W^{2}\left(\xi^{j}, \omega\right)=c\left(\xi^{j}\right) W^{1}\left(\xi^{j}, \omega\right) .
$$


Therefore, we get that

$$
L^{*}\left(\xi^{j}, \omega\right) H_{j}\left(\xi^{j}\right)=\left(h_{j 1}\left(\xi^{j}\right)+c\left(\xi^{j}\right) h_{j 2}\left(\xi^{j}\right)\right) W^{1}\left(\xi^{j}, \omega\right)=v\left(\xi^{j}\right) W^{1}\left(\xi^{j}, \omega\right),
$$

where $v\left(\xi^{j}\right)=h_{j 1}\left(\xi^{j}\right)+c\left(\xi^{j}\right) h_{j 2}\left(\xi^{j}\right)$. Notice that $W^{1}\left(\xi^{j}, \omega\right)$ and $W^{2}\left(\xi^{j}, \omega\right)$ are real vectors. Substituting (3.13) into the formula (3.12) yields

$$
\sum_{j} \int_{0}^{2 \pi}\left[T\left(\xi^{j}, \eta\right) W^{1}\left(\xi^{j}, \omega\right) \cdot W^{1}\left(\xi^{j}, \omega\right)\right]\left|v\left(\xi^{j}\right)\right|^{2} d \theta=0 .
$$

It has been shown in 14 (see the proof of Lemma 12 there) that

$$
T\left(\xi^{j}, \eta\right) W^{1}\left(\xi^{j}, \omega\right) \cdot W^{1}\left(\xi^{j}, \omega\right)>0 \quad \forall \eta,
$$

which immediately implies $v\left(\xi^{j}\right)=0$. From the asymptotic formula of $u$ (see (3.4)), we now conclude that $u(x)=O\left(|x|^{-3 / 2}\right)$.

Now we are at a position to prove the uniqueness of the outgoing radiation solution for the Dirichlet problem. As mentioned before, the same proof can be applied to other types of solutions.

Theorem 3.4. There exists at most one outgoing radiation solution for the Dirichlet problem.

Proof. It suffices to prove that if $u(x) \in H_{0}^{1,-s}(\Omega)$, which is $C^{1}$ for $|x|$ large, satisfies $\mathcal{L}^{D} u-\omega^{2} u=0$ and the radiation conditions, then $u(x) \equiv 0$ in $\Omega$. It follows from Lemma 3.3 that

$$
u(x)=o\left(|x|^{-1 / 2}\right) \quad \text { as } \quad|x| \rightarrow \infty .
$$

Since $C(x)=C$ for $|x|>R, u(x)$ is in fact $C^{\infty}$ in $\{|x|>R\}$. Let $\chi(x)=\chi(|x|) \in$ $C^{\infty}\left(\mathbb{R}^{2}\right)$ satisfy

$$
\chi(x)=\left\{\begin{array}{lll}
0 & \text { in } & |x|<5 R / 4, \\
1 & \text { in } \quad|x|>3 R / 2 .
\end{array}\right.
$$

Then $v(x)=\chi(x) u(x) \in C^{\infty}\left(\mathbb{R}^{2}\right)$ satisfies

$$
v(x)=o\left(|x|^{-1 / 2}\right) \quad \text { as } \quad|x| \rightarrow \infty
$$

and

$$
L(D, \omega)=\left(L(D)+\omega^{2} I\right) v(x)=g(x),
$$

where $\operatorname{supp}(g) \subseteq\{|x| \leq 3 R / 2\}$. Define the differential operator $L^{*}(D, \omega)$ with symbol $L^{*}(\xi, \omega)$. It is readily seen that

$$
L^{*}(D, \omega) L(D, \omega) v=\phi(D, \omega) v=L^{*}(D, \omega) g=: \tilde{g},
$$

where $\phi(D, \omega)$ is the differential operator (scalar) with symbol $\phi(\xi, \omega)$. Likewise, we have $\operatorname{supp}(\tilde{g}) \subseteq\{|x| \leq 3 R / 2\}$. Having conditions (2.3), (2.4) and the asymptotic formula (3.15) in mind, we now apply Littman's result [11, which is a generalization of Rellich's result, to conclude that

$$
u(x)=v(x)=0 \quad \text { in } \quad\{|x|>3 R / 2\} .
$$

Now by the unique continuation property, we have that $u(x)=0$ in $\Omega_{2 R}$ and thus $u(x) \equiv 0$ in $\Omega$. To use the unique continuation property, we need $u(x) \in H^{2}\left(\Omega_{2 R}\right)$, which is guaranteed by the elliptic regularity theorem with Lipschitz coefficients $C_{i j k l}(x)[7$. 


\section{LIMITING ABSORPTION PRINCIPLE}

The main theme of this paper is to prove the following theorem.

Theorem 4.1 (Limiting absorption principle). Let $\mathbb{C} \supset Q^{+}:=\left(\omega_{0}, \omega_{1}\right) \times \imath(0, \varepsilon)$, where $0<\omega_{0}<\omega_{1}$ and $\varepsilon>0$. Assume $f \in L_{c}^{2}(\Omega):=\left\{f \in L^{2}(\Omega): \operatorname{supp}(f)\right.$ is compact\}. Then the map

$$
\mathcal{R}(\cdot): Q^{+} \rightarrow H_{0}^{1,-s}(\Omega)
$$

defined by $\mathcal{R}(z) f=\left(\mathcal{L}^{D}-z\right)^{-1} f$ is uniformly continuous.

Remark 4.2. Likewise, we can prove that $\mathcal{R}(\cdot): Q^{-} \rightarrow H_{0}^{1,-s}(\Omega)$ is uniformly continuous by the same arguments, where $Q^{-}:=\left(\omega_{0}, \omega_{1}\right) \times \imath(-\varepsilon, 0)$. Also, the same conclusion holds when $\mathcal{L}^{D}$ is replaced by $\mathcal{L}^{N}$ with $H_{0}^{1,-s}(\Omega)$ being substituted by $H^{1,-s}(\Omega)$.

Theorem 4.1 will be proved below by a series of lemmas. We first need a uniform estimate of the fundamental solution $\Gamma(x, \sqrt{\zeta})$ with $\zeta \in \Lambda_{\varepsilon_{0}}^{+}=\{\zeta \in \mathbb{C}:|\zeta-\kappa|<$ $\left.\tilde{\delta}, \operatorname{Im} \zeta>\varepsilon_{0}\right\}$, where $\varepsilon_{0} \geq 0, \kappa>0$, and $\tilde{\delta}$ is sufficiently small so that $0 \notin \Lambda_{\varepsilon_{0}}^{+}$. Here $\sqrt{\zeta}$ is taken with positive imaginary part. Recall that $\Gamma(x, \sqrt{\zeta})$ is defined by

$$
\Gamma(x, \sqrt{\zeta})=\frac{1}{4 \pi^{2}} \int_{\mathbb{R}^{2}}(\zeta I-L(\xi))^{-1} e^{\imath x \xi} d \xi .
$$

Lemma 4.3. Let $\nu$ be any multi-index. Then

(i) for $\varepsilon_{0}>0, \partial^{\nu} \Gamma(x, \sqrt{\zeta})$ is a continuous function in $\mathbb{R}^{2} \backslash\{0\} \times \Lambda_{\varepsilon_{0}}^{+}$and converges to zero more rapidly than any power of $|x|^{-1}$ as $|x| \rightarrow \infty$, where the convergence is uniform in $\Lambda_{\varepsilon_{0}}^{+}$;

(ii) for $\varepsilon_{0}=0, \partial^{\nu} \Gamma(x, \sqrt{\zeta})=O\left(|x|^{-1 / 2}\right)$ as $|x| \rightarrow \infty$ uniformly for $x /|x|$ and in $\Lambda_{0}^{+}$, where $\Lambda_{0}^{+}$is defined as $\Lambda_{\varepsilon_{0}}^{+}$with $\varepsilon_{0}=0$.

Proof. This lemma can be proved by methods in [12, where the same results were shown for first order systems of partial differential equations. Here we only sketch some crucial steps and refer to [12] for more details. To establish (i), we observe that for $\varepsilon_{0}>0$

(a) $\partial_{\xi}^{\nu}(\zeta I-L(\xi))^{-1}$ is a continuous function of $(\xi, \zeta)$ in $\mathbb{R}^{2} \times \Lambda_{\varepsilon_{0}}^{+}$;

(b) for any $\xi,(\zeta I-L(\xi))^{-1}$ is an analytic function of $\zeta$ in $\Lambda_{\varepsilon_{0}}^{+}$;

(c) there exists a constant $c_{\nu}$ such that

$$
\left|\partial_{\xi}^{\nu}(\zeta I-L(\xi))^{-1}\right| \leq c_{\nu}(1+|\xi|)^{-2-|\nu|} .
$$

Then (i) is an easy consequence of Lemma 3 in 12 .

To prove (ii), we first observe that

$$
\begin{aligned}
\operatorname{det}\left(\lambda^{2}-L(\xi)\right) & =\alpha(\xi) \beta(\xi)-\gamma^{2}(\xi)-\lambda^{2}[\alpha(\xi)+\beta(\xi)]+\lambda^{4} \\
& =\left(\lambda^{2}-\lambda_{1}(\xi)^{2}\right)\left(\lambda^{2}-\lambda_{2}(\xi)^{2}\right) \\
& =\left(\lambda+\lambda_{1}(\xi)\right)\left(\lambda-\lambda_{1}(\xi)\right)\left(\lambda+\lambda_{2}(\xi)\right)\left(\lambda-\lambda_{2}(\xi)\right)
\end{aligned}
$$

where $\lambda_{j}(\xi)^{2}=\left\{(\alpha(\xi)+\beta(\xi))+(-1)^{j} \sqrt{(\alpha(\xi)-\beta(\xi))^{2}+4 \gamma^{2}(\xi)}\right\} / 2>0$ for all $\xi \neq 0$. It is clear that $\lambda_{j}(\xi)^{2}$ is homogenous of degree 2 and therefore $\lambda_{j}(\xi)(>0)$ is homogeneous of degree 1 . In view of (2.3) and (2.4), the two curves defined by $\left\{\xi \in \mathbb{R}^{2}: \lambda_{j}(\xi)=1\right\}, j=1,2$, are strictly convex and nonintersecting. Now we can 
express $\left(\lambda^{2} I-L(\xi)\right)^{-1}$ by the method of partial fraction, namely,

$$
\begin{aligned}
\left(\lambda^{2} I-L(\xi)\right)^{-1} & \\
& =\left(\operatorname{det}\left(\lambda^{2} I-L(\xi)\right)\right)^{-1} L^{*}(\xi, \lambda) \\
& =\sum_{j}\left\{\frac{P_{j}^{+}(\xi)}{\lambda-\lambda_{j}(\xi)}+\frac{P_{j}^{-}(\xi)}{\lambda+\lambda_{j}(\xi)}\right\},
\end{aligned}
$$

where $P_{j}^{ \pm}(\xi)= \pm 2 \lambda_{j}(\xi)^{-1} L^{*}\left(\xi, \lambda_{j}(\xi)\right)$ are $C^{\infty}$ functions in $\mathbb{R}^{2} \backslash\{0\}$. Therefore, we have that

$$
\Gamma(x, \sqrt{\zeta})=\frac{1}{4 \pi^{2}} \int_{\mathbb{R}^{2}} \sum_{j}\left\{\frac{P_{j}^{+}(\xi)}{\sqrt{\zeta}-\lambda_{j}(\xi)}+\frac{P_{j}^{-}(\xi)}{\sqrt{\zeta}+\lambda_{j}(\xi)}\right\} e^{\imath x \xi} d \xi
$$

The integral on the right side of (4.1) is exactly the one studied in [12. Thus, (ii) follows directly from the main theorem in [12].

Remark 4.4. As for the local behavior of $\Gamma(x, \sqrt{\zeta})$ near 0 , it is not hard to check that

$$
\Gamma(x, \sqrt{\zeta})=O(\ln |x|)
$$

and

$$
\partial^{\nu} \Gamma(x, \sqrt{\zeta})=O\left(|x|^{-1}\right) \quad \forall|\nu|=1,
$$

uniformly for $\zeta \in \Lambda_{0}^{+}$.

By means of Lemma 4.3, we can prove that

Lemma 4.5. There exist a number $R^{\prime}>0$ and a constant $c>0$ such that for all $\zeta \in \Lambda_{0}^{+}$and all $u(x) \in \mathcal{D}\left(\mathcal{L}^{D}\right)$ satisfying

$$
\mathcal{L}^{D} u-\zeta u=f \in L_{c}^{2}(\Omega),
$$

we have

$$
\|u\|_{H^{1,-s}(\Omega)} \leq c\left(\|u\|_{L^{2}\left(\Omega_{R^{\prime}}\right)}+\|f\|_{L^{2}(\Omega)}\right) .
$$

Proof. Let $\varphi \in C^{\infty}\left(\mathbb{R}^{2}\right)$ satisfy $\varphi(x)=0$ for $|x| \leq R+1 ;=1$ for $|x|>R+2$. Then

$$
\begin{aligned}
-[L(D, \sqrt{\zeta})(\varphi u)]_{i} & =[(-L(D)-\zeta I)(\varphi u)]_{i} \\
& =-\sum_{j k l} C_{i j k l}(x)\left\{\partial_{j} \partial_{l} \varphi u_{k}+\partial_{l} \varphi \partial_{j} u_{k}+\partial_{j} \varphi \partial_{l} u_{k}\right\}+\varphi f_{i} \\
& =-\sum_{j k l} C_{i j k l}\left\{\partial_{j} \partial_{l} \varphi u_{k}+\partial_{l} \varphi \partial_{j} u_{k}+\partial_{j} \varphi \partial_{l} u_{k}\right\}+\varphi f_{i} \\
& =g_{i} .
\end{aligned}
$$

The right-hand side of (4.5) has compact support. It is clear that

$$
\varphi u(x)=-\int_{\mathbb{R}^{2}} \Gamma(x-y, \sqrt{\zeta}) g(y) d y=-\int_{\mathcal{K}} \Gamma(x-y, \sqrt{\zeta}) g(y) d y,
$$

where $\mathcal{K}=\{R+1 \leq|x| \leq R+2\} \cup \operatorname{supp}(f)$ is a compact set in $\Omega$. We want to estimate

$$
\|\varphi u\|_{H^{1,-s}(\Omega)}^{2}=\int_{\Omega} \varrho^{2}\left(|\varphi u|^{2}+|\nabla(\varphi u)|^{2}\right) d x .
$$


In view of (4.6) and using (ii) of Lemma 4.3 and (4.2), we immediately have that

$$
\int_{\Omega} \varrho^{2}|\varphi u|^{2} d x \leq c_{1} \int_{\Omega}|g|^{2} d x
$$

Next, let $\mathcal{K}_{0}$ be a bounded open neighborhood of $\mathcal{K}$. Then

$$
\begin{aligned}
\int_{\Omega} \varrho^{2}|\nabla(\varphi u)|^{2} d x= & \int_{\Omega} \varrho^{2}\left|\int_{\mathcal{K}} \nabla_{x} \Gamma(x-y, \sqrt{\zeta}) g(y) d y\right|^{2} d x \\
= & \int_{\Omega \backslash \overline{\mathcal{K}_{0}}} \varrho^{2}\left|\int_{\mathcal{K}} \nabla_{y} \Gamma(x-y, \sqrt{\zeta}) g(y) d y\right|^{2} d x \\
& \quad+\int_{\mathcal{K}_{0}} \varrho^{2}\left|\int_{\mathcal{K}} \nabla_{y} \Gamma(x-y, \sqrt{\zeta}) g(y) d y\right|^{2} d x \\
= & I+I I .
\end{aligned}
$$

Using (ii) of Lemma 4.3 again, we get that

$$
I \leq c_{2} \int_{\Omega}|g|^{2} d x
$$

On the other hand, we can estimate

$$
\begin{aligned}
I I & \leq c_{3} \int_{\mathcal{K}_{0}}\left|\int_{\mathcal{K}_{0}}\right| x-\left.\left.y\right|^{-1} g(y) d y\right|^{2} d x \\
& \leq c_{3} \int_{\mathcal{K}_{0}}\left(\int_{\mathcal{K}_{0}}|g(y)|^{2}|x-y|^{-1} d y\right)\left(\int_{\mathcal{K}_{0}}|x-y|^{-1} d y\right) d x \\
& \leq c_{4} \int_{\mathcal{K}_{0}} \int_{\mathcal{K}_{0}}|g(y)|^{2}|x-y|^{-1} d x d y \\
& \leq c_{5} \int_{\mathcal{K}_{0}}|g(y)|^{2} d y \\
& \leq c_{5} \int_{\Omega}|g|^{2} d y .
\end{aligned}
$$

Combining (4.7), (4.8), (4.9), and (4.10) yields

$$
\|\varphi u\|_{H^{1,-s}(\Omega)} \leq c_{6}\|g\|_{L^{2}(\Omega)}
$$

and therefore

$\|u\|_{H^{1,-s}(\Omega)} \leq\|\varphi u\|_{H^{1,-s}(\Omega)}+\|(1-\varphi) u\|_{H^{1,-s}(\Omega)} \leq c_{7}\left(\|u\|_{H^{1}\left(\Omega_{R+2}\right)}+\|f\|_{L^{2}(\Omega)}\right)$.

Now let $\tilde{\varphi}(x) \in C_{0}^{\infty}\left(\mathbb{R}^{2}\right)$ be a real-valued function with $\operatorname{supp}(\tilde{\varphi}) \subset\{|x|<R+3\}$ and $\tilde{\varphi}(x)=1$ on $\{|x| \leq R+2\}$. Then by Korn's inequality and the strong convexity 
condition, we get that

$$
\begin{aligned}
\|\nabla u\|_{L^{2}\left(\Omega_{R+2}\right)}^{2} \leq\|\nabla(\tilde{\varphi} u)\|_{L^{2}(\Omega)}^{2} \leq c_{8} B(\tilde{\varphi} u, \tilde{\varphi} u) \\
=c_{8} \int \sum_{i j k l} C_{i j k l}(x) \partial_{l}\left(\tilde{\varphi} u_{k}\right) \overline{\partial_{j}\left(\tilde{\varphi} u_{i}\right)} d x \\
=c_{8} \int \sum_{i j k l} C_{i j k l}(x)\left\{\partial_{l} \tilde{\varphi} \partial_{j} \tilde{\varphi} u_{k} \overline{u_{j}}+\tilde{\varphi} \partial_{l} \tilde{\varphi} u_{k} \overline{\partial_{j} u_{i}}+\tilde{\varphi} \partial_{j} \tilde{\varphi} \partial_{l} u_{k} \overline{u_{i}}+\tilde{\varphi}^{2} \partial_{l} u_{k} \overline{\partial_{j} u_{i}}\right\} d x \\
=c_{8} \int \sum_{i j k l} C_{i j k l}(x) \partial_{l} \tilde{\varphi} \partial_{j} \tilde{\varphi} u_{k} \overline{u_{j}} d x+c_{8} / 2 \int \sum_{i j k l} C_{i j k l}(x) \partial_{l} u_{k} \overline{\partial_{j}\left(\tilde{\varphi}^{2} u_{i}\right)} d x \\
\quad+c_{8} / 2 \int \sum_{i j k l} \partial_{l}\left(\tilde{\varphi}^{2} u_{k}\right) \overline{C_{i j k l}(x) \partial_{j} u_{i}} d x \\
=c_{8} \int \sum_{i j k l} C_{i j k l}(x) \partial_{l} \tilde{\varphi} \partial_{j} \tilde{\varphi} u_{k} \overline{u_{j}} d x+c_{8} / 2\left(\zeta u+f, \tilde{\varphi}^{2} u\right)+c_{8} / 2\left(\tilde{\varphi}^{2} u, \zeta u+f\right) \\
\leq c_{9}\left(\|u\|_{L^{2}\left(\Omega_{R+3}\right)}^{2}+\|f\|_{L^{2}\left(\Omega_{R+3}\right)}^{2}\right) .
\end{aligned}
$$

Substituting (4.12) into (4.11) immediately gives (4.4).

Remark 4.6. Using (i) of Lemma 4.3, we can replace $\|u\|_{H^{1,-s}(\Omega)}$ by $\|u\|_{H^{1}(\Omega)}$ on the left-hand side of (4.4) and the estimate is uniform with respect to $\zeta \in \Lambda_{\varepsilon_{0}}^{+}$with $\varepsilon_{0}>0$.

Remark 4.7. Lemma 4.5 is also valid for the Neumann problem $\mathcal{L}^{N}$. The boundary data only play a role in (4.12), which is satisfied for zero Neumann data. Also, we use Korn's (second) inequality in this case. Consequently, we get an extra term $\|\tilde{\varphi} u\|_{L^{2}(\Omega)}^{2}$, but it does not affect the estimate (4.4).

Now we are ready to prove Theorem 4.1 .

Proof of Theorem 4.1. We prove this theorem by a contradictory argument. The similar approach was also used in [2] and [10]. Let the theorem be not true. Then there exist two sequences $\left\{\mu_{n}\right\},\left\{\mu_{n}^{\prime}\right\} \subset Q^{+}$such that

$$
\left|\mu_{n}-\mu_{n}^{\prime}\right|<\frac{1}{n} \quad \text { and } \quad\left\|\mathcal{R}\left(\mu_{n}\right) f-\mathcal{R}\left(\mu_{n}^{\prime}\right) f\right\|_{H^{1,-s}(\Omega)} \geq c>0 .
$$

Assume that $\mu_{n} \rightarrow \mu \in \overline{Q^{+}}$. Denote $u_{n}=\mathcal{R}\left(\mu_{n}\right) f$ and $u_{n}^{\prime}=\mathcal{R}\left(\mu_{n}^{\prime}\right) f$. Since $\mathcal{L}^{D}$ is self-adjoint, the resolvent $\mathcal{R}(\zeta)$ with $\operatorname{Im} \zeta \neq 0$ is a bounded operator in $L^{2}(\Omega)$. It is readily seen that $u_{n}, u_{n}^{\prime} \in H_{0}^{1}(\Omega) \subset H_{0}^{1,-s}(\Omega)$. We first want to claim that

$$
\sup _{n}\left\|u_{n}\right\|_{H^{1,-s}(\Omega)}<\infty .
$$

We give an indirect proof of (4.13). Suppose that (4.13) does not hold. Then there exists a subsequence of $\left\{u_{n}\right\}$, still denoted by $\left\{u_{n}\right\}$, such that

$$
\lim _{n \rightarrow \infty}\left\|u_{n}\right\|_{H^{1,-s}(\Omega)}=\infty \text {. }
$$

Define $w_{n}=u_{n} /\left\|u_{n}\right\|_{H^{1,-s}(\Omega)}$, so $\left\|w_{n}\right\|_{H^{1,-s}(\Omega)}=1$. Therefore, by Rellich's compactness theorem, there exists a subsequence of $\left\{w_{n}\right\}$, denoted by $\left\{w_{n}\right\}$ as well, such that

$$
w_{n} \rightarrow w \quad \text { in } \quad L^{2}\left(\Omega_{R_{1}}\right)
$$


Also, $w_{n}$ solves

$$
\mathcal{L}^{D} w_{n}-\mu_{n} w_{n}=f /\left\|u_{n}\right\|_{H^{1,-s}(\Omega)}=: f_{n},
$$

where $\operatorname{supp}\left(f_{n}\right)=\operatorname{supp}(f)$. By choosing $R_{1}>R^{\prime}$ with $R^{\prime}$ given in Lemma 4.5 and using Lemma 4.5, we obtain that $\left\|w_{n}-w\right\|_{H^{1,-s}(\Omega)} \rightarrow 0$ and $w \in H_{0}^{1,-s}(\Omega)$. Moreover, for any $\varphi(x) \in C_{0}^{\infty}(\Omega)$, we have that

$$
B(w, \varphi)-\mu(w, \varphi)=\lim _{n \rightarrow \infty}\left\{B\left(w_{n}, \varphi\right)-\mu_{n}\left(w_{n}, \varphi\right)\right\}=\lim _{n \rightarrow \infty}\left(f_{n}, \varphi\right)=0 .
$$

In other words, $w$ satisfies

$$
\mathcal{L}^{D} w-\mu w=0 .
$$

Now if $\operatorname{Im} \mu \neq 0$, then $\left\|w_{n}-w\right\|_{H^{1}} \rightarrow 0$ and $w \in H_{0}^{1}(\Omega)$ (see Remark 4.6), i.e. $w \in \mathcal{D}\left(\mathcal{L}^{D}\right)$. Hence $w$ must be zero. This leads to a contradiction since

$$
\|w\|_{H^{1,-s}(\Omega)}=\lim _{n \rightarrow \infty}\left\|w_{n}\right\|_{H^{1,-s}(\Omega)}=1 .
$$

On the other hand, for the case $\operatorname{Im} \mu=0$, we aim to show that $w$ satisfies the radiation conditions. To this end, let $\operatorname{supp}(f) \subset\left\{|x|<R_{2}\right\}$ for some $R_{2}>0$ and $\varsigma(x) \in C^{\infty}\left(\mathbb{R}^{2}\right)$ satisfy

$$
\varsigma(x)=\left\{\begin{array}{lll}
0 & \text { in } \quad & \left\{|x| \leq R_{2}\right\} \\
1 & \text { in } \quad\left\{|x|>R_{2}+1\right\} .
\end{array}\right.
$$

Doing the same computations as in (4.5), we can deduce that

$$
-L(D)\left(\varsigma w_{n}\right)-\mu_{n}\left(\varsigma w_{n}\right)=g\left(x, w_{n}, \nabla w_{n}\right) \text { in } \mathbb{R}^{2},
$$

where $g\left(x, w_{n}, \nabla w_{n}\right)$ contains $\partial^{\nu} w_{n}(x)$ for $|\nu| \leq 1$ and is supported in $\left\{R_{1} \leq|x| \leq\right.$ $\left.R_{1}+1\right\}=: K$ (see the right-hand side of (4.5)). Note that $g$ does not involve $f_{n}$ since $\varsigma f_{n}=0$. Therefore, $\varsigma w_{n}$ can be represented by

$$
\varsigma w_{n}(x)=-\int_{K} \Gamma\left(x-y, \sqrt{\mu_{n}}\right) g\left(y, w_{n}(y), \nabla w_{n}(y)\right) d y .
$$

Clearly, $g\left(x, w_{n}, \nabla w_{n}\right) \rightarrow g(x, w, \nabla w)$ in $L^{2}(K)$. Recall that $\Gamma\left(x, \sqrt{\mu_{n}}\right)$ converges to $\Gamma_{+}(x, \sqrt{\mu})$ uniformly in $|x|>a>0$ (see (i) of Theorem 2.1). Therefore, taking $n \rightarrow \infty$ in (4.16) gives

$$
w(x)=\varsigma w(x)=-\int_{K} \Gamma_{+}(x-y, \sqrt{\mu}) g(y, w(y), \nabla w(y)) d y
$$

for any $x$ in $\left\{|x|>R_{2}+2\right\}$. Now it is easy to see that $w(x)$ satisfies the radiation conditions (2.5). That is, $w(x)$ is an outgoing radiation solution of (4.15). By the uniqueness theorem, we obtain $w(x)=0$, which is impossible since $\|w\|_{H^{1,-s}(\Omega)}=1$. Thus, (4.13) holds. Likewise, we can show

$$
\sup _{n}\left\|u_{n}^{\prime}\right\|_{H^{1,-s}(\Omega)}<\infty .
$$

In view of (4.13), (4.17) and using Rellich's compactness theorem and Lemma4.5 again, we can prove that there exist $u, u^{\prime} \in H_{0}^{1,-s}(\Omega)$ such that

$$
\left\|u_{n}-u\right\|_{H^{1,-s}(\Omega)} \rightarrow 0 \text { and }\left\|u_{n}^{\prime}-u^{\prime}\right\|_{H^{1,-s}(\Omega)} \rightarrow 0,
$$

where, as usual, $\left\{u_{n}\right\}$ and $\left\{u_{n}^{\prime}\right\}$ represent subsequences. Going over the same arguments as above, we immediately obtain that $u$ and $u^{\prime}$ are solutions of $\mathcal{L}^{D} u-$ $\mu u=f$ and satisfy the outgoing radiation conditions if $\operatorname{Im} \mu=0$. Therefore, we 
must have $u=u^{\prime}$. But this is not possible since $\left\|u-u^{\prime}\right\|_{H^{1,-s}(\Omega)} \geq c>0$. The proof of Theorem 4.1 is now complete.

From Theorem 4.1 and its proof, we immediately have that

Corollary 4.8. There exists an outgoing (incoming) radiation solution to the exterior Dirichlet or Neumann boundary value problem for $f \in L_{c}^{2}(\Omega)$.

\section{Discussion of the SPECTRUm OF $\mathcal{L}^{D}$ AND $\mathcal{L}^{N}$}

With the help of the limiting absorption principle, we want to show that all spectrum of $\mathcal{L}^{D}$ and $\mathcal{L}^{N}$ are absolutely continuous spectrum. It suffices to prove that $\mathcal{H}_{\mathrm{ac}}^{D}=L^{2}(\Omega)$ and $\mathcal{H}_{\mathrm{ac}}^{N}=L^{2}(\Omega)$, where $\mathcal{H}_{\mathrm{ac}}^{D}$ and $\mathcal{H}_{\mathrm{ac}}^{N}$ are called the subspaces of absolute continuity of $\mathcal{L}^{D}$ and $\mathcal{L}^{N}$, respectively. We refer to 8 . for the definition and other properties of the subspace of absolute continuity.

To begin, we observe that $s\left(\mathcal{L}^{D}\right) \subset \mathbb{R}$ and $s\left(\mathcal{L}^{N}\right) \subset \mathbb{R}$ because they are selfadjoint. Here and below, $s(\cdot)$ denotes the spectrum of the operator. Now let $-\lambda<0$. Then we get from Korn's inequality that for $u \in H_{0}^{1}(\Omega)$

$$
B(u, u)+\lambda\|u\|_{L^{2}(\Omega)}^{2} \geq c \sum_{|\nu|=1}\left\|\partial^{\nu} u\right\|_{L^{2}(\Omega)}^{2}+\lambda\|u\|_{L^{2}(\Omega)}^{2} \geq \min \{c, \lambda\}\|u\|_{H^{1}(\Omega)}^{2},
$$

which implies $s\left(\mathcal{L}^{D}\right) \subset[0, \infty)$. Similarly, using Korn's inequality for $u \in H^{1}(\Omega)$, we can derive

$$
\begin{aligned}
B(u, u)+\lambda\|u\|_{L^{2}(\Omega)}^{2} & \geq \varepsilon\left(c^{\prime}\|u\|_{H^{1}(\Omega)}^{2}-c^{\prime \prime}\|u\|_{L^{2}(\Omega)}^{2}\right)+\lambda\|u\|_{L^{2}(\Omega)}^{2} \\
& =\varepsilon c^{\prime}\|u\|_{H^{1}(\Omega)}^{2}+\left(\lambda-\varepsilon c^{\prime \prime}\right)\|u\|_{L^{2}(\Omega)}^{2},
\end{aligned}
$$

where $c^{\prime}>0, c^{\prime \prime}>0$, and $0<\varepsilon<1$. Taking $\varepsilon$ sufficiently small so that $\lambda-\varepsilon c^{\prime \prime}>0$, we obtain $s\left(\mathcal{L}^{N}\right) \subset[0, \infty)$.

Theorem 5.1. $\mathcal{H}_{a c}^{D}=\mathcal{H}_{a c}^{N}=L^{2}(\Omega)$ and $s_{a c}\left(\mathcal{L}^{D}\right)=s_{a c}\left(\mathcal{L}^{N}\right)=[0, \infty)$. Here $s_{a c}(\cdot)$ denotes the absolutely continuous spectrum of the operator.

Proof. We will prove this theorem for $\mathcal{L}^{D}$. The proof for $\mathcal{L}^{N}$ is similar. To show $\mathcal{H}_{\mathrm{ac}}^{D}=L^{2}(\Omega)$, it suffices to prove that $f \in L_{c}^{2}(\Omega)$ belongs to $\mathcal{H}_{\mathrm{ac}}^{D}$ since $\mathcal{H}_{\mathrm{ac}}^{D}$ is closed in $L^{2}(\Omega)$ and $L_{c}^{2}(\Omega)$ is dense in $L^{2}(\Omega)$.

Now let $\{E(\lambda)\}$ be the spectral family for the self-adjoint operator $\mathcal{L}^{D}$. Then $E(\lambda)$ satisfies

(a) $E(\lambda)=\lim _{\varepsilon \rightarrow 0^{+}} E(\lambda+\varepsilon)=E(\lambda+0)$;

(b) $E(-0)=\lim _{\lambda \rightarrow 0^{-}} E(\lambda)=0$, for $s\left(\mathcal{L}^{D}\right) \subset[0, \infty)$;

(c) $(E(\lambda) f, f)=\|E(\lambda) f\|_{L^{2}(\Omega)}^{2} \geq 0$ is nondecreasing in $\lambda$ and

$$
\lim _{\lambda \rightarrow \infty}\|E(\lambda) f\|_{L^{2}(\Omega)}^{2}=\|E(\infty) f\|_{L^{2}(\Omega)}^{2}=\|f\|_{L^{2}(\Omega)}^{2}
$$

(see [8]). Let $\lambda>\lambda_{0}>0$. Then by Stone's formula we obtain that

$$
\left(\left[E(\lambda)-E\left(\lambda_{0}\right)\right] f, f\right)=\lim _{\varepsilon \rightarrow 0^{+}} \frac{1}{2 \pi \imath} \int_{\lambda_{0}}^{\lambda}([\mathcal{R}(z+\imath \varepsilon)-\mathcal{R}(z-\imath \varepsilon)] f, f) d z .
$$

Theorem 4.1 asserts that

$$
([\mathcal{R}(z+\imath \varepsilon)-\mathcal{R}(z-\imath \varepsilon)] f, f) \rightarrow\left(\left[\mathcal{R}^{+}(z)-\mathcal{R}^{-}(z)\right] f, f\right)
$$


uniformly on $\left[\lambda_{0}, \lambda\right]$, where $\mathcal{R}^{ \pm}(z)=\lim _{\varepsilon \rightarrow 0^{+}} \mathcal{R}(z \pm \imath \varepsilon)$. Therefore, interchanging the limit and the integral in (5.1) gives

$$
\left(\left[E(\lambda)-E\left(\lambda_{0}\right)\right] f, f\right)=\frac{1}{2 \pi \imath} \int_{\lambda_{0}}^{\lambda}\left(\left[\mathcal{R}^{+}(z)-\mathcal{R}^{-}(z)\right] f, f\right) d z .
$$

Because the left-hand side of (5.2) is nonnegative, nondecreasing, and continuous in $\lambda$, we can assume that

$$
\left(\left[\mathcal{R}^{+}(z)-\mathcal{R}^{-}(z)\right] f, f\right)=\imath G(z)
$$

for some nonnegative function $G(z)$. Taking $\lambda_{0} \rightarrow 0$ leads to

$$
(E(\lambda) f, f)=\frac{1}{2 \pi} \int_{0}^{\lambda} G(z) d z .
$$

It is clear that $G(z) \in L^{1}((0, \infty))$ because of (c). Extending $G(z)=0$ for $z<0$, we obtain that

$$
(E(\lambda) f, f)=\frac{1}{2 \pi} \int_{0}^{\lambda} G(z) d z \quad \forall \lambda \in \mathbb{R}
$$

and thus $(E(\lambda) f, f)$ is an absolutely continuous measure, i.e. $f \in \mathcal{H}_{\mathrm{ac}}^{D}$. As mentioned above, this implies $\mathcal{H}_{\mathrm{ac}}^{D}=L^{2}(\Omega)$. In other words, the subspace of singularity $\mathcal{H}_{\mathrm{s}}^{D}$ is $\{0\}$ and we have $s_{\mathrm{ac}}\left(\mathcal{L}^{D}\right)=[0, \infty)$.

Corollary 5.2. There exists no embedded eigenvalue for $\mathcal{L}^{D}$ and $\mathcal{L}^{N}$.

\section{REFERENCES}

[1] D.D. Ang, M. Ikehata, D.D. Trong and M. Yamamoto, Unique continuation for a stationary isotropic Lamé system with varaiable coefficients. Comm. in PDE, 23 (1998), 371-385. MR98j:35049

[2] K. Chelminski, The principle of limiting absorption in elasticity. Bull. Polish Acad. Sci. Math., 41 (1993), 19-30. MR97j:35144

[3] B. Dehman and L. Rabbiano, La propriété du prolongement unique pour un système elliptique: le système de Lamé. J. Math. Pures Appl., 72 (1993), 475-492. MR94h:35051

[4] G.F.D. Duff, The Cauchy problem for elastic waves in an anistropic medium. Philos. Trans. Roy. Soc. London Ser. A, 252 (1960), 249-273. MR22:2157

[5] D.M. Eidus, The principle of limiting absorption. AMS Transl., 47 (1965), 157-191. MR0188026 (32:5471)

[6] I.M. Gelfand and G.E. Shilov, Generalized Functions. Volume 1. Properties and Operations, Acedemic Press, New York, 1964. MR29:3869

[7] M. Giaquinta, Introduction to Regularity Theory for Nonlinear Elliptic Systems, Birkhäuser, Boston, 1993. MR94g:49002

[8] T. Kato, Perturbation Theory for Linear Operators, Springer-Verlag, New York, 1980. MR96a:47025

[9] V.D. Kupradze, Three-dimensional Problems of the Mathematical Theory of Elasticity and Thermoelasticity, North-Holland, Amsterdam, 1976. MR80h:73002

[10] R. Leis, Initial Boundary Value Problems in Mathematical Physics, John Wiley \& Sons Ltd. and B.G. Teubner, Stuttgart, 1986. MR87h:35003

[11] W. Littman, Decay at infinity of solutions to partial differential equations with constant coefficients. Trans. Amer. Math. Soc., 123 (1966), 449-459. MR 33:6110

[12] M. Matsumura, Uniform estimates of elementary solutions of first order systems of partial differential equations. Publ. Res. Inst. Math. Sci., 6 (1970), 293-305. MR 44:1919

[13] G. Nakamura and J.-N. Wang, Unique continuation for the two-dimensional anisotropic elasticity system and its applications to inverse problems. Submitted.

[14] D. Natroshvili, Two-dimensional steady-state oscillation problems of anisotropic elasticity. Georgian Math. J., 3 (1996), 239-262. MR97c:73017 
[15] J.R. Schulenberger and C.H. Wilcox, A Rellich uniqueness theorem for steady-state wave propagation in inhomogeneous anisotropic media. Arch. Rational Mech. Anal., 41 (1971), 18-45. MR 43:712

[16] J.R. Schulenberger and C.H. Wilcox, The limiting absorption principle and spectral theory for steady-state wave propagation in inhomogeneous anisotropic media. Arch. Rational Mech. Anal., 41 (1971), 46-65. MR 43:713

[17] N. Weck, Aussenraumaufgaben in der Theorie stationärer Schwingungen inhomogener elastischer Körper. Math. Zeit., 111 (1969), 387-398. MR41:7900

[18] N. Weck, Unique continuation for systems with Lamé principal part. Math. Methods Appl. Sci., 24 (2001), 595-605. MR2002f:35048

[19] C.H. Wilcox, Wave operators and asymptotic solutions of wave propagation problems of classical physics. Arch. Rational Mech. Anal., 22 (1966), 37-78. MR33:7675

[20] C.H. Wilcox, Steady-state wave propagation in homogeneous anisotropic media. Arch. Rational Mech. Anal., 25 (1967), 201-242. MR.37:1121

Department of Mathematics, Hokkaido University, Sapporo 060-0810, Japan

E-mail address: gnaka@math.sci.hokudai.ac.jp

Department of Mathematics, National Taiwan University, Taipei 106, Taiwan

E-mail address: jnwang@math.ntu.edu.tw 